\title{
STUDIES ON INTRAPULMONARY MIXTURE OF GASES. IV. THE SIGNIFICANCE OF THE PULMONARY EMPTYING RATE AND A SIMPLIFIED OPEN CIRCUIT MEASUREMENT OF RESIDUAL AIR
}

\author{
By ANDRE COURNAND,1 ELEANOR DEF. BALDWIN, ROBERT C. DARLING, \\ AND DICKINSON W. RICHARDS, JR. \\ (From the Department of Medicine, College of Physicians and Surgeons, Columbia University; \\ the Research Service, First Division, Welfare Hospital, Department of Hospitals; \\ and the Tuberculosis Service, Bellevue Hospital, New York City)
}

(Received for publication July 2, 1941)

In preceding papers of this series, we have studied (1) the relative effectiveness of mixing or distribution of tidal air into alveolar spaces in normal and abnormal subjects, and have proposed (2) an open circuit method for residual air determination, in which nitrogen in the lungs is washed out by continuous inhalation of pure oxygen.

The present paper (a) gives the results of a number of determinations of residual air, by the open circuit method, in normal subjects and patients with chronic cardiac or chronic pulmonary disease; $(b)$ offers certain simplifications of technique in the open circuit method; and (c) suggests further significance of some of the data which this method provides.

It will simplify the succeeding discussion if a brief review is given of the theoretical basis of the open circuit method of residual air deter- mination. The principle underlying all residual air methods which employ an inert gas, such as nitrogen or hydrogen, is the measurement of this inert gas before and after its distribution (by means of a period of breathing) between an unknown volume (the lungs) and a known volume (the spirometer). The relationship is described by a simple formula. Let nitrogen be the inert gas measured:

(Nitrogen percentage in lungs at start $\times$ lung volume $)+$ (nitrogen percentage in spirometer at start $X$ spirometer volume $)=$ (nitrogen percentage in lungs at end $X$ lung volume) + (nitrogen percentage in spirometer at end $X$ spirometer volume).

For purposes of simplification, the correction factor due to nitrogen excreted from the body (3) is here disregarded.

Solving the above equation for lung volume,

$$
\text { Lung volume }=\frac{(\mathrm{N} 2 \text { per cent in spirometer at end } \times \text { spirometer volume })}{(\mathrm{N} 2 \text { per cent in lungs at start })-(\mathrm{N} 2 \text { per cent in lungs at end })} .
$$

The factors in the numerator can be determined accurately. The major error in measurement of residual air, or functional residual air (lung volume) is due to inaccuracy in estimating nitrogen concentration in the lungs in the presence of poorly aerated lung spaces-in other words, due to inaccurate alveolar sampling (or in some methods inaccurate assumptions regarding alveolar values).

On the other hand, any errors in the final value for lung volume, due to errors in estimating alveolar nitrogen concentration, will be minimized

\footnotetext{
1 Working under a grant from the Commonwealth Fund.
}

if the denominator in the above formula can be made as large as possible. Since the alveolar nitrogen under conditions of breathing normal air is approximately the same ( $81 \pm 1$ per cent $)$, it follows that a method which brings the alveolar nitrogen, at the end of the breathing period, to a value as near zero as possible, will, other things being equal, ensure the smallest error due to stagnant air in the lungs untapped by alveolar sampling. It is this which the open circuit method provides by emptying the lungs of their nitrogen as completely as possible through continuous inhalation of pure oxygen.

In addition to giving more accurate values for 
residual air in patients with inadequate pulmonary aeration, the method permits certain estimates or approximations in alveolar values, with a minimum of error. This again is due to the large percentage difference between alveolar nitrogen at the start and that at the end of the breathing period. These approximations, providing a simplification of technique, will be discussed in a subsequent section.

\section{Results of 158 duplicate determinations of resid- ual air by the open circuit method}

On 134 subjects, 158 duplicate residual air determinations of functional residual air were made under basal conditions. Each pair of measurements was made on the same morning, with a 15- to 30-minute interval between the two tests. The results of these paired determinations show that:

In 74 duplicate tests, or 46.8 per cent of the whole series, each determination deviated by less than 2 per cent from the mean of the pair of values; in 109 duplicate tests, this deviation was less than 3 per cent from the mean; in 139 duplicates, the deviation was less than 5 per cent; and in 156 of the 158 cases, the deviation was less than 7 per cent from the mean value of the pair.

It is of interest that there is a more or less even scatter of these deviations regardless of the size of the functional residual air.

While differences larger than 5 per cent are not satisfactory from the technical standpoint, it may be noted that the cases studied include a large proportion of advanced fibrosis and emphysema. The latter, by reason of the fundamental pathology present, offer great technical difficulties for any residual air method. It is also not unlikely that some of these large percentage differences in duplicate tests were due to actual changes in residual air value through shift in pulmonary mid-position rather than to technical error. These points have been discussed in the third paper of this series (2), and will be considered again below.

The 158 duplicate determinations of residual air may be grouped according to the clinical conditions of the subjects, as follows:
17 on normal subjects;

22 on 22 patients with chronic cardiac disease in varying degrees of cardiac insufficiency; 48 on 46 patients with chronic pulmonary tuberculosis, including many with pneumothorax or thoracoplasty;

49 on 36 patients with pulmonary fibrosis or emphysema ;

22 on 22 patients with miscellaneous diseases of the chest-bronchiectasis, carcinoma of the bronchus, chest deformity, and pneumonectomy.

Figure 1 summarizes the residual air/total capacity ratio found in these five groups. The increased ratio in the emphysema group was comparable to that reported in the literature by other methods $(4,5,6)$. The ratio in our cardiac cases, however, showed less of an increase above normal than has been reported by other methods $(7,8$, $9,10)$. The 8 per cent increase of the residual air/total capacity ratio above the normal levels, in this cardiac group, is about the same as the

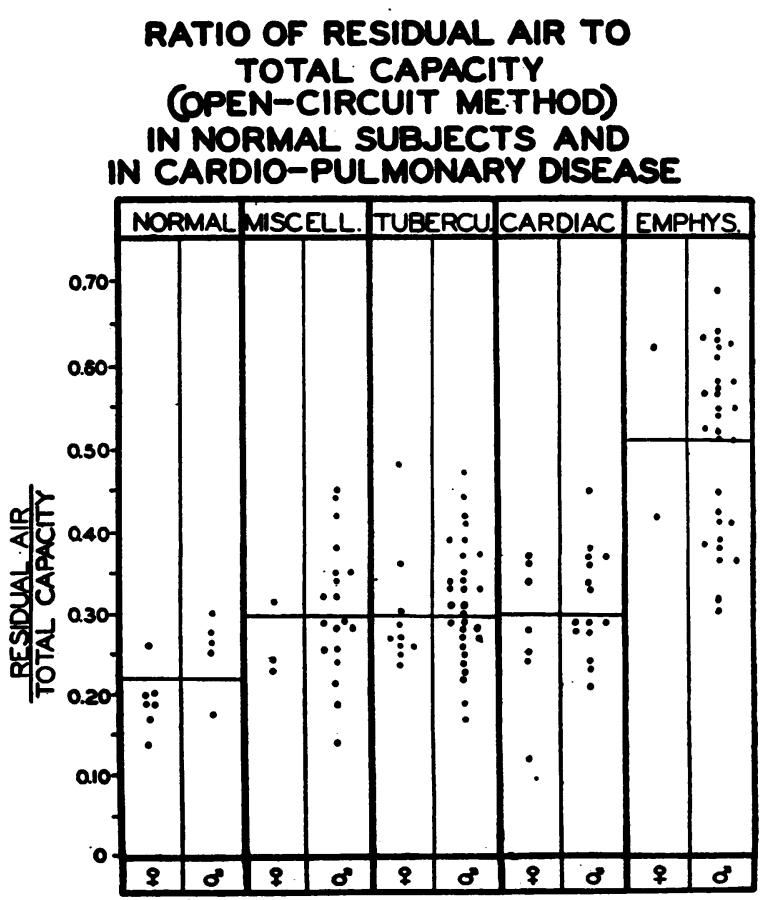

Fig. 1. Residual Air/Total Capacity Ratio in Individual Cases in Various Clinical Groups

(1) Normal subjects, (2) miscellaneous chest diseases, (3) tuberculosis, (4) cardiac disease, (5) pulmonary emphysema. 
relative increase found in the tuberculous and miscellaneous groups where there has been a shrinking of the vital capacity. A further analysis of the residual air in various types and degrees of cardiac insufficiency will be presented in a subsequent paper.

Relative constancy of the alveolar air sample taken at the start, before the beginning of pure oxygen breathing: elimination of this measurement from procedure

In Figure 2 are charted all the values for alveolar nitrogen percentage taken at rest under basal conditions while the patient breathed atmospheric air, i.e., before the beginning of pure oxygen breathing. This is the "alveolar $\bar{a}$ " sample of our earlier papers. From the data illustrated in Figure 2, it is found that 93 per cent of the alveolar $\bar{a}$ values lie between 79.50 and 82.49 per cent, with a mean value approximately 81.0 per cent. Thus, by reason of the large (alv. $\bar{a} \mathrm{~N} 2$ -alv. $\overline{\mathrm{p}} \mathrm{N} 2$ ) difference in the open circuit method (see formula above), the assumption of $81.0 \mathrm{per}$ cent as a constant for alv. $\bar{a}$ in this formula is unlikely to lead to any important error in the final value of functional residual air. We have recalculated (Figure 3) the data of the 316 residual air determinations, using 81.0 per cent as a constant value for alveolar ā nitrogen, and have found this presumption to be correct. Only 5 per cent of cases showed an alteration of more than 50 cc. in functional residual air value as a result of this recalculation. In all of these the functional residual air was over $2500 \mathrm{cc}$; ; thus the percentage error here was small. In only 2 cases was this alteration over $85 \mathrm{cc}$. (one a difference of $101 \mathrm{cc}$., the other $104 \mathrm{cc}$.). One of these latter cases was an extreme emphysema, the other a combined emphysema and cardiac case.

Thus it seems fair to conclude that the alveolar à sampling can be omitted from the open circuit technique. This shortens and simplifies the procedure. A detailed description of the revised open circuit technique is given at the end of the paper.

Alveolar air sample taken at the end of the period of pure oxygen breathing: its usefulness as a measure of pulmonary emptying rate, and the physiological significance of this function

According to the open circuit procedure, the subject, under basal conditions, breathes pure oxygen for 7 minutes, thus washing out the nitrogen in the pulmonary air spaces. This expired air is

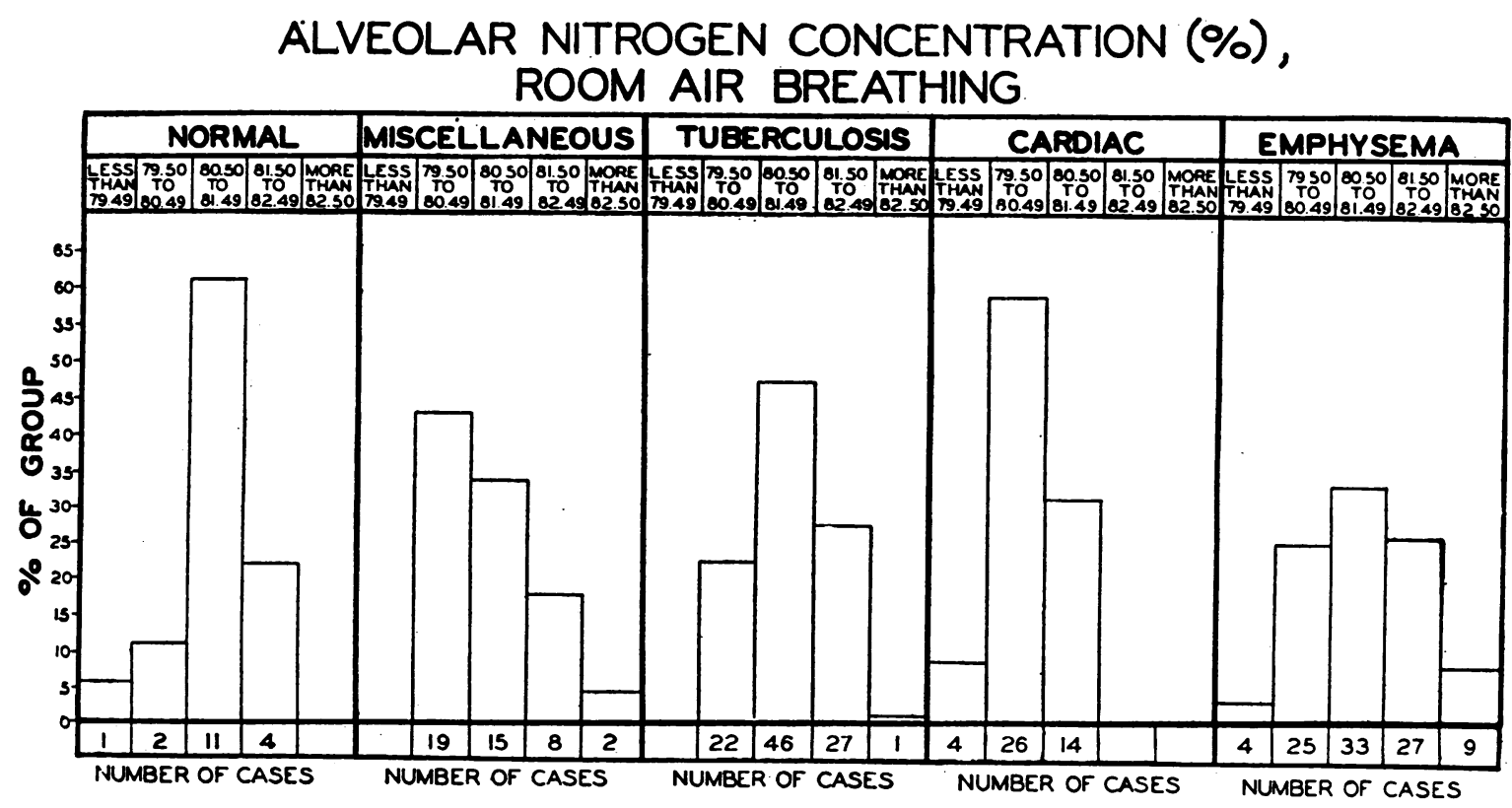

Fig. 2. Values of Alveolar Nitrogen Concentration, with the Subjects Breathing Atmospheric Air, in Various Clinical Conditions 
ERROR IN FUNCTIONAL RISIDUAL AIR DUE TO THE ASSUMPTION OF 81.00\% AS THE STANDAND VALUE FOR ALVEOLAR $T$

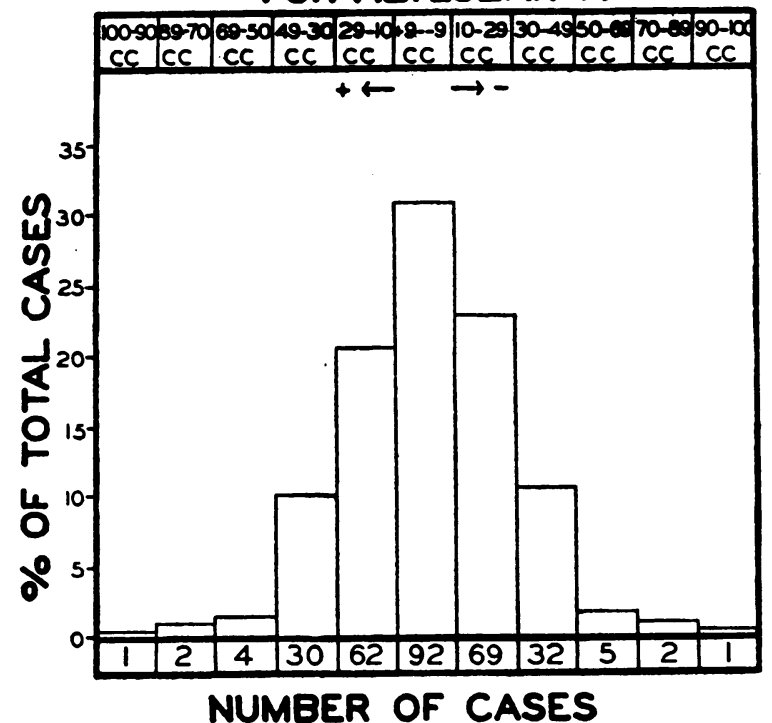

Fig. 3. The Extent of ERror in the Value of Residual Air (Open Circuit Method) Caused by Assuming 81.0 Per Cent as the Normal Alveolar Nitrogen Concentration, as Compared with Residual Air Values Calculated from Alveolar Nitrogen ConCentrations Measured by Experiment

collected and subsequently analyzed for nitrogen content. At the end of the 7 minutes of oxygen breathing, the subject exhales completely (into a separate circuit) and a sample of expired (alveolar) air is taken into an evacuated sampling tube.

The procedure of washing out the lungs by oxygen breathing, considered from the purely ventilatory standpoint, is almost ideal for the purpose of demonstrating the effectiveness of distribution of tidal air through alveolar spaces during quiet breathing, since it is only through the adequate distribution of inhaled pure oxygen into the pulmonary spaces that the nitrogen contained in them can be displaced and exhaled.

Thus the completeness of the washing-out of nitrogen from the lungs by a stated period of pure oxygen breathing (which we have termed the pulmonary emptying rate) can be considered an effective measure of distribution of tidal air through alveolar spaces. In an efficiently ventilated lung, therefore, an alveolar specimen taken at the end of a relatively short period of oxygen breathing should show a low nitrogen concentration; in an inefficiently ventilated lung, the nitrogen concentration of this specimen should be high.

This concept is corroborated by the analysis of the data covering "alveolar $\overline{\mathrm{p}}$ " nitrogen measurements in 316 residual air determinations, as illustrated in Figure 4.

If one takes as an upper limit an "alveolar $\overline{\mathrm{p}}$ " nitrogen of 2.5 per cent, one finds that 94 per cent of the determinations in normal subjects, and 100 per cent of those on cardiac patients, are well below the level; whereas above it are 17.7 per cent of tuberculous patients, 17 per cent of the miscellaneous group, and 97 per cent of the emphysema patients.

The difference in performance between the normal and the cardiac groups, on the one hand, and the groups with organic pulmonary disorders, on the other, is thus well defined, though perhaps not unexpected.

It will be apparent that pulmonary emptying, or effectiveness of ventilation, depends in great part upon certain obvious anatomical and physiological relationships. A subject, for example, with small functional residual air, whose tidal air is large and respiratory rate rapid, will, other things being equal, tend to empty his pulmonary spaces rapidly. The emptying that will be expected to occur in a given time with a known functional residual air, effective tidal air, and rate of respiration, and assuming perfect intrapulmonary mixing, can be easily calculated by the usual compound interest equation (the interest rate having here a negative value). In most emphysematous subjects, the large ratio of residual air to tidal air is an important factor in delayed emptying. In addition, however, there is the factor of unequal distribution of tidal air, certain large regions of the lungs being often very inadequately ventilated. This factor is more difficult to express quantitatively, but in advanced pulmonary fibrosis and emphysema is usually the dominant influence in producing delayed pulmonary emptying. A striking evidence of this tendency to inadequate ventilation is shown in Figure 4, where practically all cases of pulmonary fibrosis and emphysema show a decreased rate of pulmonary emptying, including those subjects with relatively small residual airs as well as those with large. 

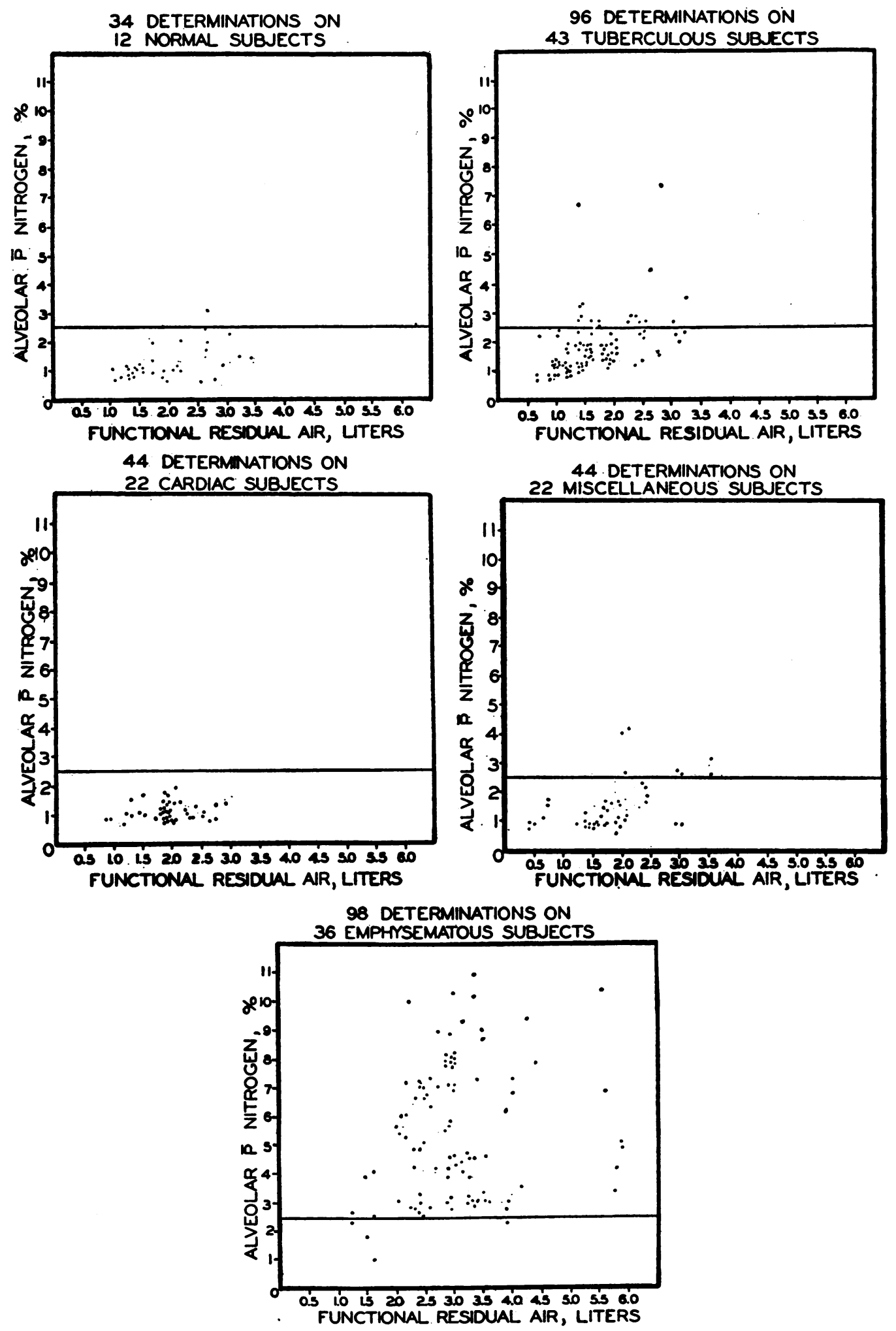

Fig. 4. "Pulmonary Emptying Rate"-Alveolar Nitrogen Concentration at the End of 7 Minutes of Pure Oxygen Breathing in 156 Duplicate Determinations in Various Clinical Conditions, as Related to the Size of the Functional Residual Air 
Arterial oxygen unsaturation, which was also studied in a number of cases (Figure 5), was only partially correlated with pulmonary emptying, low saturation occurring at times with rapid emptying, and normal saturation with slow emptying. This is not unexpected, as there may be all degrees of vascular perfusion of poorly aerated regions of the lungs.

Of considerable interest to us was the consistent performance of the group of cardiac patients in their effective pulmonary emptying. None of these, it is true, had very large residual air values, but it seemed theoretically not unlikely that the "lungenstarre" of pulmonary congestion might interfere with effective aeration of some of the perfused pulmonary air spaces. Such was evidently not the case in any of the patients whom we studied, as far as this particular criterion of pulmonary emptying indicated. Objection might be raised that the low alveolar nitrogen values were due to poor sampling, since these patients often had small reserve air volumes. The fact, however, that alveolar carbon dioxide levels were comparable in these samples with arterial carbon dioxide tensions eliminates this objection. That hyperventilation, which was definite in most cases, may have played a part in the low alveolar $\bar{p}$ nitrogen percentages is entirely possible, but this does not alter the fact of effective pulmonary emptying in every case under the conditions of the test. Since some of the cardiac patients had a moderate arterial anoxemia, between 89 and 91 per cent, it is suggested that there were other mechanisms for this than inadequate ventilation of perfused alveolar spaces; i.e., perfusion of regions completely unventilated (shunting) or other factors removing air from immediate contact with blood such as by dilatation and congestion of alveolar

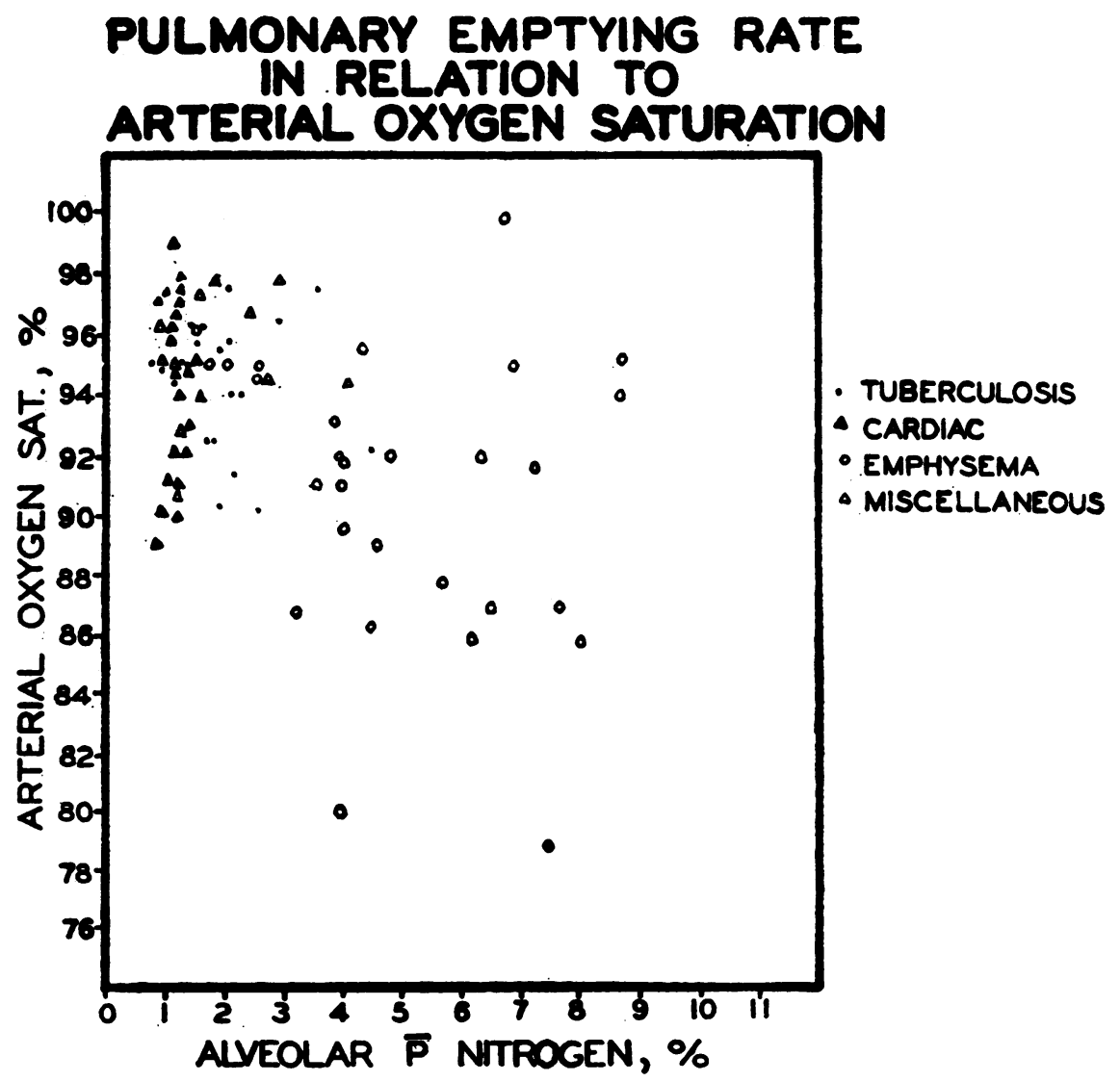

Fig. 5. Relation between Arterial Oxygen Saturation and Pulmonary Emptying Rate in Various Clinical Conditions

“Miscellaneous" refers to a group of miscellaneous chest conditions (see text). 
capillaries, or edema, or thickened, less permeable alveolo-capillary walls-in other words, defective diffusion of gases through fluids, as distinct from defective distribution of gases through air spaces.

At this point it should probably be emphasized that this particular test of "pulmonary emptying "- the alveolar nitrogen after 7 minutes of oxygen breathing-is in certain respects quite arbitrary. It tends, as a matter of fact, to demonstrate particularly defects in emptying under conditions found in pulmonary emphysema with relatively large residual air values. Under other conditions, a 3-minute or even a 1-minute period of oxygen breathing will more readily demonstrate slight degrees of defective intrapulmonary mixing. Also, under certain conditions, the small amount of nitrogen excreted from the body during oxygen breathing becomes a significant factor in the alveolar $\bar{p}$ value. An exhaustive analysis of this question has been undertaken by one of the present authors (R. C. D.) and will be reported separately. It is probably fair, however, to consider the pulmonary emptying rate, above described, as an approximate clinical index of effectiveness of ventilation. As an index of pathological intrapulmonary mixing, the pulmonary emptying rate has to be considered along with other factors-functional residual air, tidal air, and rate of respiration.

\section{"Anatomical" versus "physiological" residual air volumes}

Reference to Figure 4 shows that, in a considerable number of cases of advanced emphysema, the alveolar $\bar{p}$ nitrogen percentage is very high, up to 8 or 10 per cent or even higher. In other words, 7 minutes of oxygen breathing has not been able to remove more than 90 per cent of the alveolar air originally present in the lungs. The question naturally arises whether in such instances there may not be stagnant or very poorly ventilated lung spaces which remain high in nitrogen after oxygen breathing, but whose contents cannot be adequately exhaled into an "alveolar" air sample, the true mean alveolar nitrogen being actually higher than that obtained. The value obtained for residual air in these cases would therefore be erroneous. As pointed out in the third paper of this series (2), this excessive stagnation of intrapulmonary air can sometimes be proved by prolonging the time of pure oxygen breathing to 10 or 12 minutes, and a more accurate residual air determination can be obtained in this way.

The ultimate in pulmonary stagnation probably occurs in certain cases of large positive-pressure bulla formation. There is one such case in the present series, a man of 40 with an expanding bulla occupying two-thirds of the left thoracic cavity and displacing the mediastinum to the right. Residual air determination by the open circuit method revealed a very small functional residual air volume, and a rapid and complete pulmonary emptying with low alveolar $\bar{p}$ nitrogen. The interpretation of these findings is clear: the large bulla was inactive in pulmonary function; in fact completely isolated from the working pulmonary mechanism, except for the small increment of inspiratory air that must have been drawn into the bulla in order to maintain its positive pressure. This case, incidentally, is the only one in the emphysema group that showed a pulmonary emptying rate appreciably below 2.5 per cent (Figure 4).

The distinction between physiologically ventilating, pathologically ventilating, and non-ventilating pulmonary air spaces, is an interesting one. In the last named of these, the separation between physiological and anatomical residual air volumes becomes practically complete.

The open circuit method of residual air measurement, when combined with the alveolar $\bar{p}$ nitrogen, or pulmonary emptying rate, thus provides more than a value for static or anatomical residual lung volume. It furnishes an index of mixing, or effective distribution, of tidal air through the alveolar spaces during quiet breathing. When these measurements are included with the values for the other divisions of lung volume and with those of total resting pulmonary ventilation and respiratory rate, the purely ventilatory aspect of pulmonary function at rest can be analyzed with a fair degree of completeness. We have discussed elsewhere (11) the dual classification of pulmonary function into ventilatory (air displacement) and respiratory (respiratory gas exchange). 
Revised technique of open circuit method for residual air 2

The apparatus used ${ }^{3}$ has been described in the third paper of this series (2). It consists essentially of three parts: (a) a standard 100-liter Tissot gasometer; $(b)$ an open circuit, with the inspiratory tubing leading from an anesthesia bag (which in turn is kept partly filled with oxygen from a tank) to the mouthpiece, and the expiratory tubing from mouthpiece to gasometer; $(c)$ a second open circuit, to which the subject can be connected by turning the respiratory valve at the mouthpiece, with expiratory tubing leading out past evacuated sampling tubes for alveolar air sampling.

One change has been made in the apparatus: A long vertical drum revolving at constant rate has been attached to the side of the Tissot gasometer. A pen attached to the counterweight of the spirometer records on this drum each expiratory movement, thus affording a measure of respiratory rate and expiratory amplitude.

Procedure. In a preliminary test, the external lung volumes-tidal air, vital capacity, complemental and reserve air-are recorded on a spirograph. This has the added advantage of training the subject in breathing procedures. After a 15-minute rest period, the subject, under basal conditions and lying supine except for a two- or three-pillow head and shoulder elevation, is attached to the mouthpiece, with the valve turned to the second circuit so that he is inhaling outside air. (The main circuit and gasometer have previously been thoroughly washed with oxygen by 6 successive fillings and emptyings of the Tissot gasometer with 15 to 20 liters of oxygen through the entrance bag.) With the gasometer empty, volume and temperature readings are taken. Oxygen flow at approximately the (volume) rate of the subject's resting ventilation is then started from the tank. Precisely at the subject's expiratory level, just before inspiration begins, the valve is turned to shift the subject's breathing into the oxygen circuit. The subject then breathes oxygen quietly for 7 minutes. (At a convenient time during this interval, the second of the respiratory valves at the mouthpiece is turned, thus shutting off the inspiratory tubing of the second [atmospheric air] circuit. This permits an uncontaminated alveolar sampling later. In subjects with a small reserve air, such as cardiac patients, it is practical to wash out the second circuit with oxygen during this interval.) At the end

$2 \mathrm{~A}$ footnote in the third paper of this series indicated that the authors were planning a simplification of the method in which no alveolar samples would be necessary. While such a method could be used with approximate accuracy in almost all cases, we have retained the "alveolar $\overline{\mathrm{p}}$ " nitrogen measurement because of the importance and independent significance which this measurement affords.

3 The apparatus was made by the Warren E. Collins Company, Boston, Massachusetts. The special 5-way respiratory valve was designed and constructed for us by Mr. Herman P. Roth of the Warren E. Collins Company. of the inspiratory phase nearest the exact moment of termination of the 7-minute period, the valve is turned, shifting the subject from first to second circuit, and he is instructed to exhale completely, thus furnishing the alveolar $\overline{\mathbf{p}}$ sample which is taken in the evacuated sampling tube. The subject may then be disconnected from the apparatus. The reason the valve is turned at peak of inspiration is to provide a larger volume of air for washing out the second circuit for the alveolar sample. The amount of nitrogen contained in this tidal air is negligible, so that its loss from the total expired air in the gasometer will cause no error. Finally, the main circuit is flushed with about 10 liters of oxygen into the gasometer, so as to wash any remaining nitrogen out of the inflow tubing. The second volume and temperature readings on the gasometer are recorded, and the outflow tubing is washed through promptly with the air from the gasometer. Two samples are taken from the gasometer for analysis.

The gasometer and main circuit are washed out with oxygen, as before; 15 to 30 minutes are allowed for the subject's lungs to return to conditions of room air breathing; and the determination is then repeated.

Gas analyses are all now carried out by the use of the Van Slyke-Neill apparatus. Certain minor alterations in the procedure as described by Peters and Van Slyke (12) have been kindly communicated to us by Dr. N. L . Kaltreider.

The calculation of functional residual air is as follows:

$$
\begin{aligned}
& F . R . A . \text { (dry) }=\frac{(V+D S)(N S-N O)}{81-\text { alv. } N}-C . \\
& \text { F.R.A. (dry) = functional residual air in cc. (calculated } \\
& \text { as dry gas). } \\
& V \quad=\text { volume reading of spirometer gas, cor- } \\
& \text { DS rected to } 37^{\circ} \text { (dry). } \\
& \text { DS } \quad=\text { volume of dead space under spirometer, }
\end{aligned}
$$

The final figure for functional residual air volume is computed from F.R.A. (dry) by correcting for water vapor saturation, as exists (approximately) within the lungs.

$$
\text { F.R.A. }=\text { F.R.A. }(\text { dry }) \times \frac{\text { Barometer pressure }}{\text { Barometer pressure }-48} \text {. }
$$

\section{SUM MARY}

1. The nitrogen concentration of a sample of alveolar air taken at the end of a period of pure oxygen breathing provides an index of pulmonary 
emptying which is useful as a measure of effectiveness of ventilation.

2. This procedure detects delayed pulmonary emptying, particularly in cases of pulmonary fibrosis and emphysema. Patients with such conditions have alveolar nitrogen concentrations, after 7 minutes of oxygen breathing, above 2.5 per cent; normal subjects and patients with chronic heart disease have values below 2.5 per cent.

3. One hundred and fifty-eight determinations of residual air by the open circuit method are presented and analyzed.

4. A revised and somewhat simplified method for open circuit determination of residual air, with simultaneous measurement of pulmonary emptying, is described.

\section{BIBLIOGRAPHY}

1. Cournand, A., Darling, R. C., Mansfield, J. S., and Richards, D. W., Jr., Studies on the intrapulmonary mixture of gases. II. Analysis of the rebreathing method (closed circuit) for measuring residual air. J. Clin. Invest., 1940, 19, 599.

2. Darling, R. C., Cournand, A., and Richards, D. W., Jr., Studies on the intrapulmonary mixture of gases. III. An open circuit method for measuring residual air. J. Clin. Invest., 1940, 19, 609.

3. Darling, R. C., Cournand, A., Mansfield, J. S., and Richards, D. W., Jr., Studies on the intrapulmo- nary mixture of gases. I. Nitrogen elimination from blood and body tissues during high oxygen breathing. J. Clin. Invest., 1940, 19, 591.

4. Lundsgaard, C., and Schierbeck, K., Untersuchungen über die Volumina der Lungen. Acta Med. Scandinav., 1923, 58, 495.

5. Anthony, A. J., Untersuchungen über Lungenvolumina und Lungenventilation. Deutsches Arch. f. klin. Med., 1930, 167, 129.

6. Hurtado, A., Fray, W. W., and McCann, W. S., Studies of total pulmonary capacity and its subdivisions. IV. Preliminary observations on cases of pulmonary emphysema and of pneumoconiosis. J. Clin. Invest., 1933, 12, 833.

7. Lundsgaard, C., Determination and interpretation of changes in lung volumes in certain heart lesions. J. A. M. A., 1923, 80, 163.

8. Binger, C. A. L., The lung volume in heart disease. J. Exper. Med., 1923, 38, 445.

9. Christie, R. V., and Meakins, J. C., Intrapleural pressure in congestive heart failure and its clinical significance. J. Clin. Invest., 1934, 13, 323.

10. Altschule, M. D., The pathological physiology of chronic cardiac decompensation. Medicine, 1938, $17,75$.

11. Cournand, A., and Richards, D. W., Jr., Forms of pulmonary insufficiency. I. Discussion of a physiological classification and presentation of clinical tests. Amer. Rev. Tuberc., 1941, 44, 26.

12. Peters, J. P., and Van Slyke, D. D., Quantitative Clinical Chemistry. Vol. II. Methods. Williams and Wilkins., Baltimore, 1932.

13. Cournand, A., and Riley, R. L. (To be published.) 